\title{
SINGLE-CRYSTAL X-RAY DIFFRACTOMETRY USING SYNCHROTRON RADIATION
}

\author{
K. EICIIIIORN AND K. HÜMMER \\ Institut für Kristallographie, Universität (TII) Karlsruhe \\ Taiserstraße 12, 76128 Karlsrule, Germany
}

The outstanding properties of synchrotron radiation, in particular its high brilliancy over a wide spectral range, its low divergence, its polarization properties, and the pulsed time structure, extend the range of single-crystal $\mathrm{X}$-ray difractometry to experiments which are not feasible with conventional sources, such as scaled X-ray tubes or rotating anode equipment. Data collection techniques are strongly infuenced by the general aims of a diffraction experiment, by the sample quality, its absorption and scattering power, as well as by the reflection profile shape and the instrumental resolution function. Often, the sample propertics play a crucial role, and not all samples may be suitable for data collection with synchrotron X-rays. The time-dependence of the primary beam intensity and of its polarization state requires monitoring and normalization to monitor counts, which complicates data collection and data reduction due to sources of both random and systematic errors not known from conventional X-ray sources. There is almost no utilization of X-ray diffraction that cannot profit from the use of synchrotron radiation. X-ray difraction at a synchirotion radiation source can yield structure factors of an unprecedented quality, provided proper attention is given to sample properties, to data collection strategy and data evaluation procedures. Though little is gained for strong reflections, the improvement is very pronounced for the weaker reflections, including high-order reflections, which can be measured in much shorter time than with conventional X-ray sources. However, synchirotron racliation does not provide a solution to all problems, in some cases conventional laboratory $\mathrm{X}$-ray sources may be more appropriate than synchrotron radiation. Taking into account the limited access to synchrotron radiation sources, $\mathrm{X}$-ray diffraction with synchrotron radiation can only supplement, but not replace conventional $X$-ray sources and diffraction techniques.

PACS numbers: $07.85 .+\mathrm{n}, 61.10 . \mathrm{Jv}$

\section{Introduction}

Single-crystal X-ray diffraction with synchrotron radiation has meanwhile become a well-established branch of X-ray crystallography which profits from the 
high intensity over a wide spectral range, a small source size and a low divergence, the polarization properties, and the pulsed time structure of a synchrotron radiation source.

In the following we will try to give an overview of the subject, which is inevitably biased by our own past experiences with synchrotron radiation. We will first present a short review of $\mathrm{X}$-ray scattering by crystal structures and of the basics of single-crystal X-ray diffractometry. We will then discuss the shape of reflection profiles obtained with synchrotron $\mathrm{X}$-rays and the crucial role played by the sample quality. Step scan and data collection techniques, as well as some of the problems specific to experimentation with synchrotron radiation will be reviewed and we will conclude with an overview of current applications and an outlook into the possible future of single-crystal X-ray diffractometry with synchrotron radiation.

\section{X-ray scattering by single crystals}

For single crystals, whose atoms are arranged on a 3-dimensional periodic lattice, we observe interference effects similar to those of an optical grating. Constructive interference and intensity enlancement is observed only when the scattering vector $\boldsymbol{H}$, defined by the momentum transfer from incident $\left(s_{0}\right)$ to diffracted ray $(s)$

$$
H=\frac{s-s_{0}}{\lambda}
$$

coincides with a reciprocal lattice vector

$$
\boldsymbol{H}=h \boldsymbol{a}^{*}+k \boldsymbol{b}^{*}+l \boldsymbol{c}^{*} \text {. }
$$

$h, k, l$ are the indices of lattice planes with spacing $d=1 /|H| ; a^{*}, b^{*}, c^{*}$ are the basis vectors of the reciprocal lattice, and $\lambda$ is the $X$-ray wavelength. The geometric conditions to observe diffraction are given by the Laue equations

$$
\begin{aligned}
& \left(s-s_{0}\right) \cdot a=h \cdot \lambda, \\
& \left(s-s_{0}\right) \cdot b=k \cdot \lambda, \\
& \left(s-s_{0}\right) \cdot c=l \cdot \lambda .
\end{aligned}
$$

$\mathrm{X}$-ray diffraction by single crystals may formally be described as a reflection from lattice planes $h, k, l$, with $\boldsymbol{H}$ being normal to these planes. The angle $2 \theta$ between incident and diffracted ray is given by Bragg's law ( $n$ is the diffraction order)

$$
n \lambda=2 d \sin \theta \text {. }
$$

An ideal single crystal can be described as the convolution of the electron density distribution $\rho_{c}(v)$ in the unit ccll with the crystal lattice, represented mathematically by a 3-dimensional periodic array of delta functions ( $u, v, w$ integers)

$$
\rho(r)=\rho_{\mathrm{c}}(r) \sum_{u, v, w} \delta(r-u a-v b-w c) .
$$

In the kinematical theory diffraction from such an array is described by its Fourier transform, according to the convolution theorem by the product of the Fourier 
transform of the array of delta functions with the Fourier transform of the clectron density in the unit cell

$$
\mathcal{F}[\rho(\boldsymbol{v})]=\mathcal{F}\left[\rho_{\mathrm{c}}(\boldsymbol{v})\right] \mathcal{F}\left[\sum_{u, v, w} \delta(u, v, w)\right]=F(\boldsymbol{H}) G(\boldsymbol{H})
$$

The lattice factor $G(H)$ describes the shape of a reflection; the structure factor $\boldsymbol{F}(\boldsymbol{H})$ contains the superposition of the waves scattered from within the unit cell

$$
\boldsymbol{F}(\boldsymbol{H})=\sum_{j} f_{j} \exp \left[2 \pi \mathrm{i} r_{j} \boldsymbol{H}\right] .
$$

The sum extends over all the atoms at coordinates $r_{j}$ in the unit cell; $f_{j}$ is the so-called formfactor of the $j$-th atom (including the effect of thermal vibrations)

$$
f=f_{0}+f^{\prime}+\mathrm{i} f^{\prime \prime} \text {. }
$$

It is the Fourier transform of the atomic density distribution $\rho_{\mathrm{a}}(\boldsymbol{v})$

$$
f_{0}(S)=\int \rho_{\mathrm{a}}(\boldsymbol{r}) \exp [2 \pi \mathrm{i} r \boldsymbol{S}] \mathrm{d} V
$$

normalized to the scattering of one free electron. It decreases with increasing momentum transfer $|S|$. The terms $f^{\prime}$ and $f^{\prime \prime}$ are called "dispersion corrections", which are often small, but in the order of several electrons close to an absorption edge.

The structure factor $F(H)$ of the unit cell is, in general, a complex number (real for centrosymmetric crystal structures), defined by both its amplitude and its phase angle

$$
\begin{aligned}
& \boldsymbol{F}(\boldsymbol{H})=A(\boldsymbol{H})+\mathrm{i} B(\boldsymbol{H}), \\
& \tan \phi(\boldsymbol{H})=B(\boldsymbol{H}) / A(\boldsymbol{H}) .
\end{aligned}
$$

From the properties of the Fourier transform it follows that the inverse transform $\mathcal{F}^{-1}[\rho(r)]$ gives

$$
\rho(\boldsymbol{r})=\frac{1}{V} \sum_{\boldsymbol{H}} F(\boldsymbol{H}) \exp [-2 \pi \mathrm{i} \boldsymbol{r} \boldsymbol{H}],
$$

the electron density in the unit cell ( $V$ is the unit cell volume). There are two important points to remember:

1. Elastically scattered X-ray intensities $I(H)$ carry information about the details of the electron density distribution in the unit cell.

2. Since we measure $I(H) \propto|F(H)|^{2}$, only the modulus $|F(H)|$ of the structure factor can be directly obtained. Its phase $\phi(\boldsymbol{H})$, however, is initially unknown ("phase problem").

\section{Single-crystal X-ray diffractometry}

The aim of an X-ray diffraction experiment is to obtain structure factor amplitudes $|F(H)|$ for reflections $h, k, l$; sometimes only for a limited number of reflections, but more often data sets complete up to some maximum $\sin \theta / \lambda$ (corresponding to some minimum observed lattice spacing $\left.d_{\min }=\lambda / 2 \sin \theta_{\max }\right)$. The 
observed structure amplitude $|F(H)|$ is related to the integrated intensity $I(H)$ (total scattered energy when the sample is rotated through the diffracting position); $I \propto|F|^{2}$ in the kinematical theory. Determination of $|F(H)|$ involves measurement of $I(\boldsymbol{H})$ as accurately as possible, and - particularly important with the limited beam time at synchrotron radiation sources - as efficiently and as rapidly as practicable.

Bragg's law suggests two ways of performing a diffraction experiment:

1. variation of the X-ray energy at fixed angle 0: energy-dispersive technique;

2. variation of the angle $\theta$ at a fixed $X$-ray energy: angle-dispersive technique. The latter is the method routinely used for single-crystal X-ray diffractometry which will be discussed in the following.

In order to observe diffraction from a single crystal the photon counter has to be positioned under the correct Bragg angle 20, and the sample must then be oriented such that the reciprocal lattice vector $H$ is in the plane of incident and diffracted rays, $s_{0}$ and $s$, defined by both source and detector, with the lattice planes $h, k, l$ bisecting the angle between $s_{0}$ and $s$.

Several diffraction geometries are in use for this purpose, the two most common being the 4-circle diffractometer and the kappa-axis goniometer; each one has 3 circles for rotations to orient the crystal, plus the detector $2 \theta$-axis (see e.g. $[1,2])$. The Euler cradle is still the most popular crystal orienter which combines excellent mechanical rigidity and stability (important for experiments which require ovens, cryostats, pressure cells, etc.) with only minor restrictions on sample orientation. The also popular liappa geometry offers a greater accessibility of the sample environment at the expense of weight that can be supported. Since data collection techniques are almost identical for both geometries and since setting angles are easily transformed between the two geometries, we will focus on the 4 -circle geometry in the following.

The setting angles $\omega, \chi, \phi$ (or $\omega^{\prime}, \kappa, \phi^{\prime}$ for kappa-axes) required to observe a particular reflection $h, k, l$ depend on the sample orientation which is specified by the product $U \cdot B$ of the two $3 \times 3$-matrices $U$ and $B ; B$ is a matrix that transforms from generally triclinic reciprocal axes to a set of Cartesian axes, and $U$ is an orthogonal $3 \times 3$ rotation matrix which brings these Cartesian axes into coincidence with a laboratory frame rigidly attached to the diffractometer circles. Details depend on the choice of this laboratory frame and on the sense of rotations and their zero points, but in gencral

$$
\left(\begin{array}{l}
x \\
y \\
z
\end{array}\right)=U B\left(\begin{array}{l}
h \\
k \\
l
\end{array}\right)
$$

transforms from indices $h, k, l$ to Carlesian coordinates $x, y, z$ referred to the laboratory frame. To put a reciprocal lattice point into the reflecting position, the sample is then rotated through Euler angles $\omega, \chi, \phi$

$$
h_{\theta}=\Omega X \Phi \cdot U B \cdot h
$$

$(\Omega, X, \Phi$ are the corresponding rotation matrices) such that both incident and difracted ray are correctly positioned in the equatorial plane of the detector 20 -circle. 
In principle, there is an infinite number of solutions for each reflection $h, k, l$, corresponding to azimuthal rotation around the scattering vector $\boldsymbol{H}$ (so-called $\psi$-rotation). That particular position where the reciprocal lattice vector $\boldsymbol{H}$ coincides with the plane of the Eulcr cradle is called the bisecting position; the latter is routincly used for data collection $(\psi=0)$.

The orientation matrix $U B$ is usually refined from obscrved angles of a series of centered reflections. Since

$$
(U B)^{\mathrm{T}} U B=B^{\mathrm{T}} B=G^{*},
$$

where $G^{*}$ is the reciprocal metric tensor with elements $G_{i j}=a_{i}^{*} a_{j}^{*}$, refined cell constants and their e.s.d.'s are casily obtained from the $U B$-matrix.

In order to measure an integrated intensity $I(h k l)$ we need to rotate the sample crystal and to record the intensity of the diffracted ray as the reciprocal lattice point passes through the Ewald sphere

$$
R=\int_{-\Delta \omega}^{+\Delta \omega} I(\omega) \mathrm{d} \omega
$$

since the reflection contributes to intensity over a small and finite angular range required to put all mosaic blocks into the reflecting position.

The essential steps of a standard data collection are:

- determination of the sample orientation;

- sample characterization, including its quality and reflection profile shape;

- choice of data collection parameters such as aperture size, scan mode, step size and scan width, an $h k l$-order that minimizes motor slewing times, and the counting time per step;

- actual data collection, including a rapid data reduction and analysis to allow feedback into the running measurement.

Today's diffractometer-software eliminates a lot of manual effort and the tedious details of a data collection. It cannot, however, substitute a careful planning and a thoughtful execution of a diffraction experiment.

\section{Reflection profile shape}

\subsection{Resolution function}

From the incident whitc-radiation spectrum a small monochromatic band is selected using suitable single crystals. According to Bragg's law

$$
n \lambda=2 d \sin 0
$$

a single-crystal monochromator will not only transmit the wavelength $\lambda$, but also its higher harmonics $\lambda / n, n=2,3, \ldots$ (unless they are "forbidden"). Higher harmonics may be suppressed by (slightly) detuning a double-crystal arrangement which, in addition, allows for a constant beam exit. Typically, perfect $\mathrm{Si}$ and $\mathrm{Ge}$ crystals are being used for monochromatization, which are stable in the intense white X-ray beam, yiclding an cnergy resolution $\Delta E / E \approx 10^{-4}$, and an intrinsic width of the reflection curve (Darwin width) in the order of seconds of arc - for 
Si(111) e.g. $\Delta \theta_{\mathrm{D}}=0.036 \mathrm{mrad}$ at $8 \mathrm{keV}$ - which is well matched to the natural divergence of synchrotron $X$-rays.

An important advantage of synchrotron $X$-rays over the characteristic radiation from anode materials of a sealed tube is the absence of the $\alpha_{1}-\alpha_{2}$-splitting, yielding much simpler (unimodal) reflection profiles with a small wavelength dispersion. Therefore, an $\omega$-scan is the scan teclnique to be used for intensity data collection rather than $\omega-20$ scans frequently used with conventional diffractometers. Such an $\omega$-scan integrates - for each step - over the detector aperture, and results in a 1-dimensional reflection profile (rocking curve) which is the projection of the 3-dimensional reflection shape onto the $\omega$-axis.

An $\omega$-scan yields the best possible resolution for a given diffractometer; each reflection must be measured individually, however. More efficient are area detectors which allow a fairly rapid two- or even three-dimensional exploration of reciprocal space. For a 2-dimensional analysis of reciprocal space with single point-counters the so-called $Q$-scan may be used whose start and endpoint are defined by arbitrary coordinates $(h, k, l)_{\text {start }}$ and $(h, k, l)_{\text {end }}$, with steps $(\Delta h, \Delta k, \Delta l)$. Such a scan is made with a combination of the four diffractometer angles, without any fixed relationship between the individual circles. In special directions (radially) $Q$-scans may degenerate to pure $\omega-2 \theta$ scans.

The 3-dimensional shape of a Bragg peak is determined by the instrumental resolution function and by the sample, combining the effects from beam divergence, wavelength-bandpass, sample- and monochromator mosaicity, and sample size. For collection of integrated reflection intensities the projection of this resolution function onto the scan axis $(\omega)$ is required to define the correct scan range and step size. Usually the resolution function is approximated by a convolution of Gaussians [3]. This approach simplifies calculations but the final result tends to obscure the influence of the individual parameters. Much more useful for practical purposes is an approach which considers the spatial orientation of the individual contributions from beam, sample and instrument to the diffractometer's resolution function (e.g. $[4,5])$. Usually the individual components have different orientations in the instrument's angle space, and it is easier that way to identify components which contribute to the resolution function in a specific direction. This is of particular importance when an optimum resolution is required for some experimental problem.

Neglecting the contribution from the sample, the instrumental resolution function does not depend on azimuthal rotation, i.e. it is independent of the $\psi$-value. This is not always the case, however, when sample properties are taken into account, in particular not for disordered and twinned crystals, for which reflection profile slape may strongly depend on sample orientation.

\subsection{Reflection profiles}

The projection of the 3-dimensional resolution function on the scan axis must be known in order to determine the width for each scan. The resolution function is even more important when details of the three-dimensional diffraction pattern are to be examined. Assuming a convolution of Gaussian components, the full 
width at half maximum (FWIIM) of the rocking curve (this is the angular range over which a crystal diffracts as it is rotated through the Bragg position) has the following major contributions [1]:

$$
\Delta \theta^{2}=\left(\Delta 0_{\mathrm{S}}^{2}+\Delta \theta_{\mathrm{Q}}^{2}+\Delta \theta_{\mathrm{C}}^{2}\right)+\Delta \theta_{\mathrm{M}}^{2}+\Delta 0_{\lambda}^{2},
$$

$$
\begin{array}{ll}
\Delta \theta_{\mathrm{S}} & : \text { natural divergence of the SR beam }\left(\propto \lambda^{0.4} / E\right), \\
\Delta \theta_{\mathrm{Q}}=\sigma_{\mathrm{Q}} / R & : \text { angle subtended by the source at the sample, } \\
\Delta \theta_{\mathrm{C}}=\sigma_{\mathrm{C}} / R & : \text { angle subtended by the sample at the source, } \\
\Delta 0_{\mathrm{M}} & : \text { mosaic spread of the crystal, } \\
\Delta \theta_{\lambda}=(\Delta \lambda / \lambda) \tan 0 & : \text { wavelength dispersion spread }\left(\propto \lambda^{2}\right),
\end{array}
$$

where $\sigma_{\mathrm{Q}}$ is the source size, $\sigma_{\mathrm{C}}$ is the crystal size, and $R$ is the source-to-sample distance. This means, divergence is determined by the source, by geometric factors, by the wavelength bandpass, and by the sample properties. Collecting $\theta$-dependent terms, the above equation reduces to the well-known expression

$$
\Delta 0^{2}=A^{2}+B^{2} \tan ^{2} 0 .
$$

The natural divergence of the synchrotron radiation $\Delta 0_{\mathrm{S}} \approx 1 / \gamma(\gamma=1957 E$, where $E$ is the electron beam energy in $\mathrm{GeV}$ ) is of the order of the Darwin width of a Bragg reflection from perfect crystals (polarization neglected)

$$
\Delta \theta_{\mathrm{D}}=\frac{2 r_{\mathrm{e}}}{\pi V} \frac{\lambda^{2}}{\sin 20}\left|F_{\mathrm{H}}\right|=\frac{4 r_{\mathrm{e}}}{\pi V} d_{1 \mathrm{II}}^{2}\left|F_{\mathrm{II}}\right| \tan \theta .
$$

The bandwidth (dispersion spread) $\Delta \lambda / \lambda=\Delta E / E$ of a perfect-crystal monochromator is, neglecting $\Delta \theta_{\mathrm{Q}}$ and $\Delta \theta_{\mathrm{S}}$,

$$
\Delta \lambda / \lambda=\cot \theta \cdot \Delta \theta_{\mathrm{D}}
$$

as can be seen by differentiating Bragg's law. Typical values for the above quantities are:

$$
\begin{array}{llll}
\Delta \theta_{\mathrm{S}} \approx 0.10 \mathrm{mrad} & \left(0.005^{\circ}\right), & \Delta \theta_{\mathrm{M}} & \left(0.01-0.03^{\circ}\right), \\
\Delta \theta_{\mathrm{Q}} \approx 0.03 \mathrm{mrad} & \left(0.002^{\circ}\right), & \Delta \theta_{\mathrm{D}} \approx 0.03 \mathrm{mrad} & \left(0.002^{\circ}\right), \\
\Delta \theta_{\mathrm{C}} \approx 0.02 \mathrm{mrad} & \left(0.002^{\circ}\right), & \Delta \lambda / \lambda=4 \times 10^{-4} & (\text { Si } 1,1,1) .
\end{array}
$$

Due to the small source size $(\leq 1 \mathrm{~mm})$, the small vertical beam divergence $(\approx 0.1 \mathrm{mrad})$, and the large source-to-sample distance $(30-40 \mathrm{~m}$ ) the full width at half maximum (FWIIM) of the rocking curve is mainly determined by the sample, not by the synchrotron X-rays. The FWIIM of mosaic crystals is almost exclusively determined by their mosaic spread $0_{\mathrm{M}}$; for perfect crystals both beam divergence and Darwin width must be taken into account. Perfect crystals have a FWHM $\Delta 0 \leq 0.01^{\circ}$; reflections from good quality mosaic crystals have a FWHM of typically $0.02-0.03^{\circ}$, which is about a tenth of what one observes with a sealed $\mathrm{X}$-ray tube. For comparison: a sealed tube with a $150 \mathrm{~mm}$ long $0.5 \mathrm{~mm}$ diameter collimator will give a divergence of about $3 \mathrm{mrad}$, corresponding to $0.2^{\circ}$ FWHM.

The above discussion applies only to the verlical plane for which the natural divergence $\Delta \theta_{\mathrm{S}}$ of the synchrotron $\mathrm{X}$-rays is extraordinarily small; the divergence in the storage ring plane is - for a bending magnet - given by the length $l$ of 
the segment of the electron beam which can be seen by the sample at a distance $R$ :

$$
\Delta 0_{\mathrm{h}}=l / R \text {. }
$$

This quantity is defined by slits and compares well with the divergence of conventional $\mathrm{X}$-ray sources. The result is an extremely anisotropic resolution function with excellent resolution $\left(\leq 10^{-4} \AA^{-1}\right)$ in the vertical plane, and an order of magnitude smaller in the horizontal plane.

Summarizing, we can state that single-crystal diffractometers at a synchrotron radiation source can provide, without additional experimental effort, a resolution that compares to triple-axis spectrometers, without intensity losses, with an excellent peak-to-background ratio. This allows high-resolution X-ray diffractometry with short data collection times, even for the weaker reflections.

\subsection{Influence of the sample quality}

As we have seen, the profile width and shape of mosaic crystals is determined by their mosaic spread, not by the synchrotron X-ray beam divergence. The sharp reflection profiles from good-quality mosaic crystals result in a much improved peak-to-background ratio, a very welcome feature. 'This is not so, however, for poor-quality samples: the highly collimated beam makes the sample quality readily apparent. The distribution of mosaic blocks is often very inhomogeneous; rocking curves from poor-quality samples comprise several, more or less well-separated peaks, often combined with a highly anisotropic peak width and shape which depends on sample orientation. These effects interfere with measurement of accurate reflection intensities; they allow a sample characterization and investigation of twinning, domains, and disordered crystals, however.

Measurement of an integrated intensity may thus not always be a trivial task; it may require modified scan techniques, such as continuous scan mode (discussed further below) to ensure complete integration over the sample volume and wide detector apertures at the expense of increased background. Most importantly, the volume that actually scatters at each point of a step scan is only part of the total sample volume, resulting in appreciable intensity losses. The question how much intensity can be gained relative to a sealed $\mathrm{X}$-ray tube is therefore not so easy to answer. A simple statement of photon flux at the sample position may be highly misleading. The actual intensity gain depends on the sample; it is larger for perfect crystals and for crystals with a small mosaic spread (on the 4-circle machine at IIASYLAB intensity gain is typically 1 to 2 orders of magnitude). In the worst case it may turn out impossible to measure true integrated intensities for all reflections of a sample with highly anisotropic mosaic spread (no such problem might be encountered for the same sample using sealed X-ray tubes) and the peak intensity may be, for a given sample, smaller at a synchrotron radiation source than with $\mathrm{X}$-rays from a sealed tube.

The intrinsically high resolution may be a nuisance for intensity data collection on bad-quality samples, but it is a welcome property for sample characterization, particular in cases of twimned and disordered crystals. 


\subsection{Influence of the deleclor aperlure}

So far we have neglected the influence of the detector on the resolution function, making the assumption that the whole reflected beam actually enters into the detector - a very important requirement for mcasurement of integrated reflection intensities. Otherwise, an additional detector term had to be added to the above equations. A small receiving slit or - even better - an additional analyzer crystal between sample and detector is very useful, however, to further increase resolution, at the expense of intensity. Integrated intensities may become meaningless in this latter case because of scan truncation crrors.

\section{Step scan modes}

In cases where $\Delta \lambda / \lambda($ or $\Delta d / d)$ is the dominant contribution to the reflection width (e.g. the $\alpha_{1}-\alpha_{2}$ splitting of a scaled X-ray tube), an $\omega-20$-scan is appropriate with a detector aperture wide enough to accept the whole diffracted beam, incorporating geometric factors and broadening from mosaic spread. For synchrotron radiation and perfect-crystal monochromators the wavelength spread is very small $\left(\Delta \lambda / \lambda=4 \times 10^{-4}\right.$ for $\mathrm{Si}(111)$ at $\left.8 \mathrm{keV}\right)$, unless strain causes a variation of $\Delta d / d$.

To measure an integrated reflection intensity $I(h k l)$ the sample has to be rotated through a limited angular range, required to sweep the reciprocal lattice node $h, k, l$ and the sample's mosaic blocks through the reflecting position, while the detector counts the diffracted photons. Since the incident radiation is strictly monochromatic, we have a distinct 20 (from Bragg's law) for each $d$-value. With the low-divergence $X$-ray beam the reflection profile closely rescmbles the mosaic spread (see above), and an $\omega$-scan is appropriate for data collection, not an $\omega-20$-scan ( $\omega$-20-scans are required, howcver, to locate reflections when the wavelength is not exactly known). Any detector movement may move the reflection out of the detector opening, cutting into the tails of a peak. This may still yield nice-looking profiles with apparently low background, but it also yields systematically wrong intensities. The detector opening must be wide enough to intercept the whole diffracted beam, allowing for errors in the orienting matrix used to calculate setting angles $20, \omega, \chi, \phi$. Errors in these angles will offset the reflection from the center of the detector slits; comparison of calculated and centered peak positions may give an idea of the order of magnitude of these errors (for a good $U B$-matrix setting angles may be accurate to few steps in the difractometer circles).

Many crystals have reflection profiles with sharp peak and long tails. A traditional step scan requires therefore, due to the small step size, a large number of steps to safely reach the background, yet to sample the peak region with sufficient accuracy to determine a reliable integrated intensity. Actually, an appreciable amount of measuring time is then "wasted" on measuring the tails and the background. A better distribution between peak and tails may be achieved by using spread step scans with finer steps in the peak center, and wider steps in the tails $[6,7]$. Such unequal step-widtl scans require, however, that the peak position is precisely known.

To further reduce the number of profile sampling points, and the amount of output, a continuous scan mode may be applied with counter read-out "on the 
fly" at fixed time intervals. This provides a true integration over the peak, and a small number of "steps" is sufficient to sample the peak profile. Since each step is actually an integral, this may create problems with the dead time correction for higher count rates. This is certainly a drawback of the continuous scan method, but it works fine for weaker and medium intensity reflections, tolerating peak offsets of $0.01^{\circ}$ and even larger, relaxing the requirements on the accuracy of the orientation matrix $U B$. Continuous scan mode is often the best scan type to choose for poor-quality samples with split reflections and anisotropic peak shapes, but it is also valuable for fast data collection on high-quality crystals.

Continuous scan mode is also the optimum scan technique to use with automatic reflection search procedures. The narrow width of reflection profiles interferes with traditional peak search techniques, as the narrow peaks are easily missed in a (coarse) step scan. Knowledge of the instrumental resolution function may help to optimize step sizes for an automatic search ("peak hunt"). The step width in 20 depends only on the detector aperture size, $\chi$ is rather insensitive and may step $1-2^{\circ}$ at a time, but $\omega$ and $\phi$ may require very fine step widths. The solution is to use either a continuous scan mode or to apply film teclniques to bracket the search range for subsequent scans.

Determination of the sample orientation is rather straightforward if the cell constants and the setting angles of a couple of reflections are already known. Otherwise, the quickest method is often to take a rotation photograph, using photographic film or an image plate. Reflection positions are read off the developed film or image plate and the diffractometer is instructed to search and center a couple of these film reflections. Reflection search may be combined with an auto-indexing procedure; under favorable conditions a refined orienting matrix is obtained in less than an hour.

Knowledge of the cell constants and of the indices of a few intense low-order reflections simplifies the orienting procedure. This knowledge may easily be obtained from conventional powder diflraction prior to a synchrotron radiation experiment.

\section{Experimental problems}

\subsection{Monitorizalion of intensily and polarizalion}

Since the beam intensity decays during a "fill", it is essential to monitor the beam intensity $I_{0}$ in order to normalize diffracted intensities. There are several methods; the two most common are to use ionization chambers or the scattering from amorphous materials inserted in the beam. At IIASYLAB we use a "polarimeter" [8] which simultancously monitors intensity $I_{0}$ and degrce of linear polarization $Q$ of the incident bcam,

$$
I_{0}=I_{\mathrm{h}}+I_{\mathrm{v}} \text { and } Q=\left(I_{\mathrm{h}}-I_{\mathrm{v}}\right) / I_{0},
$$

$I_{\mathrm{h}}$ and $I_{\mathrm{v}}$ being the intensities of horizontally and vertically linear polarized beam components, respectively.

$Q$ depends on the position of the sample relative to the electron orbit and may change during a fill. Typically, $I_{v} \approx 0.05 I_{\mathrm{h}}$, giving $Q \approx 90 \%$. Errors in the 
assessment of the degree of polarization are much less dramatic than might be expected: for medium Bragg angles between $30^{\circ} \leq 20 \leq 50^{\circ}$ and complete neglect of the polarization correction even a change of $Q$ from $90 \%$ to $80 \%$ would affect the integral intensity by only about $2 \%[9]$.

The polarization factor derived from $Q$ is

$$
P=\frac{1}{2}\left[(1+Q)+(1-Q) \cos ^{2} 20\right]
$$

assuming a vertical diffraction gcometry where we have almost no intensity loss through polarization factors.

\subsection{Normalizalion}

The time-dependence of the $\mathrm{X}$-ray source intensity requires a decay correction and normalization of the measured reflection intensities. The individual counts are therefore scaled to a constant (reference) monitor count with counting statistics and dead time of the monitors taken properly into account (see below).

For each profile count $C_{i}, i=1, \ldots, n$ there are additional counts from beam monitors $C_{\mathrm{h} i}$ and $C_{\mathrm{v} i}$. Defining a local beam intensity $I_{i}=\left(C_{\mathrm{h} i}+C_{\mathrm{v} i}\right)$ and a polarization ratio $Q_{i}=\left(C_{\mathrm{hi}}-C_{\mathrm{v} i}\right) / I_{i}$, the simplest approach would be to scale each step count $C_{i}$ for its local $I_{i}$ and $Q_{i}$ individually. This however, has a serious disadvantage: for all but the weaker reflections the accuracy of the data would then be determined by the monitor counts rather than by the reflection shape and intensity. $A$ better approach is to use a verages $\left\langle C_{\mathrm{l}_{\mathrm{l}}}\right\rangle$ and $\left\langle C_{\mathrm{v}}\right\rangle$ : averaging improves statistics by a factor $\sqrt{n}$, where $n$ is the number of profile points. Since $n$ is typically about 100 , the error can thus be reduced by an order of magnitude relative to the simple point-to-point normalization approach.

Therefore, our data normalization strategy looks like follows [9]: normalization to $\left(\left\langle C_{\mathrm{h}}\right\rangle+\left\langle C_{\mathrm{v}}\right\rangle\right)$ provided both $C_{\mathrm{h} i}$ and $C_{\mathrm{v} i}$ do not show a significant drift or scatter; otherwise we try to correct with a least squares fit to $\left(C_{\mathrm{h} i}+C_{\mathrm{v} i}\right)$ before a veraging. If such a correction turns out insufficient, a point-to-point normalization is applied. Since the contribution from the monitors to the standard deviation of the integrated intensity, $\sigma(I)$, is taken into account, "bad" reflections are automatically given a larger standard deviation than "good" reflections and a (much) smaller weight in subsequent data processing and structure refinement.

\subsection{Counler dead lime}

An important point to consider is the effect of higher harmonics and dead time losses in the counting chains (dead time is the time following an event during which no further events can be detected). Data reduction includes a dead time correction for all the photon counters, including monitors.

For a pulsed source (like a storage ring) the time structure of the source may complicate dead time correction at high count rates [7], which are therefore avoided by inserting appropriate combinations of attenuation filters. For a dead time shorter than the bunch separation nothing is gained by decreasing the dead time, unless it could be reduced below the bunch length (in the order of nanoseconds). A dead time longer than the bunch separation makes the counter unresponsive for several bunches. Actually, bunch frequency is in the order of MIIz, count 
rates are often $\leq 10^{5}$, i.e. on a verage only one out of several hundred bunches may lead to a detectable event, even for the stronger reflections. $\mathrm{NaI}(\mathrm{Tl})$ scintillation counters have dead times of a few $\mu$ s (limited by the decay of light emission); for such a detector a storage ring appears like a continuous $\mathrm{X}$-ray source (this may not be the case for the much faster plastic scintillators), and the usual correction (e.g. $[10,11])$ applies

$$
N_{\text {corr }}=N_{\text {win }} /\left(1-\tau N_{\text {int }}\right) \text {. }
$$

This correction has to consider the lolal count rate $N_{\text {int }}$, including higher harmonics. $N_{\text {win }}$ is the count rate in the single-clannel analyzer (SCA) window, set to reject higher harmonics. Since $N_{\text {win }}$ is only a part of the total count rate, the often used approximation

$$
N_{\text {corr }}=N_{\text {win }} /\left(1-\tau N_{\text {win }}\right)
$$

is simply wrong. Assuming that $N_{\text {int }} / N_{\text {win }}=K$, i.e. a constant ratio of zero order to higher harmonics, we may write

$$
N_{\text {corr }}=N_{\text {win }} /\left(1-\tau K N_{\text {win }}\right)=N_{\text {win }} /\left(1-\tau^{\prime} N_{\text {win }}\right)
$$

using an "effective dead time" $\tau^{\prime}=K \tau$. For practical purposes this approximation has been found to work satisfactorily, provided the relative higher-harmonics content does not change significantly during data collection [9]. The preferred method, however, is to measure both zero order and higher harmonics simultaneously and to use $N_{\text {int }}$ for dead time correction [12].

Since the separation of two bunclies $(<1 \mu \mathrm{s})$ is smaller than the dead time of a $\mathrm{NaI}(\mathrm{Tl})$ counting chain, electronics cannot separate two or more photons per bunch; several photons per bunch will give - due to pile-up - a pulse of a higher energy, which is rejected by the SCA.

A proper choice of monochromator crystals may help to reduce the higher-harmonics content: the sccond order reflection 2,2,2 of the 1,1,1 reflection from Si or Ge is "forbidden"; the allowed $3,3,3$ and $4,4,4$ can be discriminated against electronically using pulse-height discrimination (SCA). Additional detuning of the double-crystal monochromator reduces the higher harmonics contamination to $I(\lambda / 3) / I(\lambda) \leq 10^{-3}$.

\section{Data collection}

There is no simple and gencral answer to the question how to collect single-crystal X-ray diffraction data with synchrotron radiation. Some of the problems related to data collection with synchrotron radiation have been discussed by Wendschul-Josties and Wulf [13] and by Kirfel and Eichhorn [9]. The latter deal with high precision structure factor measurement, the former address aspects of data collection on small and/or poor-quality crystals (though the authors do not state this explicitly).

In general, one wants to collect a data set, complete to some maximum $\sin \theta / \lambda$-value, of sufficient quality in the shortest possible time. The details of data collection depend on the gencral aims of the experiment, the synchrotron radiation spectrum, sample quality, sample absorption and scattering power, peak width and shape, and both absorption and parasitic scatter in air. Most of these factors are 
wavelength-dependent and partly counteractive (e.g. a decreasing integral reflectivity with decreasing wavelength, but smaller absorption, smaller Darwin width and generally a better peak-to-background ratio at shorter wavelengths). The optimum wavelength for some given conditions is not always obvious and sometimes difficult to determine. So far, there exists no clear cut answer, and further investigations are still required on this subject.

For each reflection wc want optimize scan width and step size, counting time per step, and we need to determine the proper filter combination. The narrow reflection profiles from samples with a small mosaic spread require very small steps for measuring, typically $0.002-0.003^{\circ}$, in order to allow for a proper integration. The accuracy of calculated angular positions is, for good quality crystals, after refinement of the orienting matrix, in the order of $0.005-0.010^{\circ}$. For poor-quality samples, however, position crrors may easily exceed $0.010^{\circ}$. Since the peak position is therefore not always accuratcly known, a pre-scan may be required to locate the peak center, to select attemuation filters, and to set the counting time per step for an optimized main scan. Thercfore, the data collection algorithm implemented on IIASYLAB's 4-circle and kappa-axis diffractometers [12] comprises the following main features:

- location of the reflection position, if required by making a pre-scan,

- determination of the proper filter combination, if required,

- evaluation of the optimum scan parameters, and

- a subsequent step scan with real-time monitoring of the beam intensity and polarization for data reduction.

The usual procedure to obtain a higher accuracy is to extend the counting time per profile step. If we do so at the synchrotron source we will, however, encounter a significant decay of primary beam intensity over the reflection profile; the decay rate depends on the lifetime of the electron beam in the storage ring. Such a decay requires a correction; in the worst case we are back to a point-per-point scaling, loosing the factor $\sqrt{n}$ in accuracy. This leads to the paradox situation that increasing of the measurcment time may actually decrease the data quality. To obtain data of higher accuracy it is therefore generally preferred to measure symmetrically equivalent reflections and to improve data by a veraging rather than to extend the counting time to more than 1-2 s/step. An alternative is to scan each reflection repeatedly and to add scan profiles until the desired accuracy has been reached. Typically, we use $0.1-1 \mathrm{~s} / \mathrm{step}$ for data collection.

An important part of data collection is the repcated measurement of a set of test reflections at regular intervals. Statistical analysis of the test reflections may give a first hint on data quality and yiclds a measure of beam and sample stability and an opportunity to derive time-dependent scaling functions to put all reflections on the same (relative) scale, e.g. in the case of a crystal decay in the $\mathrm{X}$-ray beam. It is common practice to check the intensity of the standards vs. time, and to correct for fluctuations, if required. In principle one might check for a dependence on various experimental parameters; such checks are, however, rarely done. 
The time-dependence of the synchrotron radiation source may easily create time-dependent errors not known from experiments with sealed X-ray tubes, which may pass unnoticed without an extra analysis. Improper treatment may easily defeat the advantages gained by high intensity and low divergence of the synchrotron radiation source. Data evaluation should therefore include additional checks to make sure there are no such errors left uncorrected. The simplest and quickest method is to plot the intensity of the test reflections vs. the monitor count rate; ideally such a plot cxhibits only random scatter.

\section{Applications}

Diffraction experiments with synchrotron radiation make use of the high intensity, the good collimation, the polarization properties, and the wavelength tunability over a fairly wide spectral range.

The high photon flux permits measurement of weak effects such as diffuse scattering, magnetic and nuclear scattering, and satellite reflections. The low divergence yields a high intrinsic collimation, high resolution and a good signal-to-noise ratio. Thus features close to the main Bragg peaks and from twinned crystals can be resolved.

The high source intensity makes experiments possible that require a small sample volume, e.g. the use of high-pressure cells. Data collection is possible on crystals down to and below $10 \mu \mathrm{m}$ diameter; essentially crystal structure analysis becomes feasible using a single powder grain.

Measurement of weak and very weak ("forbidden") reflections profits from high intensity, low divergence, almost 0 -independent polarization, and a good peak-to-background ratio. Data collection at short wavelengths is useful to decrease both absorption and extinction effects and provides the resolution required for high precision structure analysis.

Wavelength tunability is frequently used to exploit resonant X-ray scattering ("anomalous dispersion") for structure research. Examples are determination of absolute configuration, contrast variation, and phase determination from both single and multiple-wavelength measurements, and the investigation of structural details involving the anomalous scatterer(s).

$\mathrm{X}$-ray dichroism and double refraction is observed in the vicinity of absorption edges, causing an anisotropy and polarization dependence of anomalous scattering. This anisotropy may give rise to a violation of extinction rules for glide-planes and screw-axes, with orientation- and polarization-dependent intensities. More recently, these effects have been successfully used to derive (partial) phase information.

Phase information on triplet-phase relationships can also be derived from multiple-beam diffraction ("Umweganregung"), providing an experimental solution of the well-known "phase problem" in X-ray crystallography.

Other applications are both resonant and non-resonant magnetic X-ray scattering and time-resolved $\mathrm{X}$-ray diflraction. The former profits from high intensity; tunability and polarization properties, the latter exploits the time structure of the synchrotron radiation source. 


\section{Future developments}

Traditionally, monochromatic-beam, small-molecule X-ray diffractometry has been using $\mathrm{NaI}(\mathrm{Tl})$ point detectors; area detectors - in particular image plates [14] - have come recently into an increasing use. Image plates provide a large dynamic range $\left(1: 10^{5}\right)$, a good linearity, a low background and high sensitivity for low count rates, and reusability. They can be expected to replace the scintillation counters of single-crystal diffractometers for routine data collection, in particular for rapid measurcment of large amounts of diffraction data, using the well-established rotation method [15]. Replacement of diffractometers by image plate systems with on-line scanners is increasingly common for protein crystallography for which rapid data collection is crucial as many macromolecules suffer radiation damage in the $\mathrm{X}$-ray beam.

The image plate is ideally suited to gaining a quick overview over (parts of) reciprocal space; the extended view provided by an image plate (similar to photographic film) is very convenient for a preliminary investigation of sample quality, systematic absences, superstructure peaks, twinning, incommensurability, etc.

This raises the question about the future of single-crystal diffractometry at synchrotron radiation sources, where beam time is of prime importance. From the limited material that is a vailable at present it appears that data collection with image plates may yield structure amplitudes of a quality comparable to conventional diffractometry; image-plate data probably have a quality sufficient for high precision work like electron density analysis, but this has to await more rigorous tests in the future. So far data collection and data reduction techniques have mainly been optimized for protein crystallography; small-molecule crystallography may require some modification of the data evaluation procedures.

A problem with image plates is certainly the limited high-angle resolution of the presently available on-line systems which may be overcome by using a diffractometer in combination with off-line scanners. Controlled movement of the plate during exposure may serve to extend its applicability to time-resolved studies. Even the use of both scintillation counters and image plates on the same machine may be a sensible choice for certain diffraction experiments.

\section{Conclusions}

Synchrotron radiation has a pronounced impact on scattering experiments in general, and on X-ray diffraction in particular. Crystallographers are using synchrotron radiation facilities for expcriments that take advantage of the outstanding characteristics of synchrotron radiation, namely, a wide distribution of wavelengths, high intensity, low divergence, lincar polarization, and a pulsed time structure. There is hardly any field of X-ray diffraction that cannot profit from the use of synchrotron radiation. Single-crystal X-ray diffraction with synchrotron radiation may liclp to solve traditional problems; synchrotron radiation may also help to extend the range of $X$-ray diffractometry to experiments which are not feasible with conventional $X$-ray sources and traditional equipment. In addition to the more familiar diffraction experiments new types of crystallographic studies are progressing rapidly with more general access to synchrotron radiation sources. 
Synchrotron radiation does not provide a solution to all problems, in some cases conventional laboratory X-ray sources may be more appropriate than synchrotron radiation. Taking into account the limited access to synchrotron radiation sources, X-ray diffraction with synchrotron radiation can only supplement, but not replace conventional $X$-ray sources and diffraction techniques. Ilowever, single-crystal X-ray diffraction with synchrotron radiation can be expected to remain in the forefront of crystallographic research in the ncxt several years.

\section{Acknowledgments}

Financial support by the Bundesminister für Forschung und Technologie is gratefully acknowledged.

\section{References}

[1] U.W. Arndt, B.T.M. Willis, Single Crystal Diffractometry, Cambridge University Press, Cambridge 1966.

[2] H.L. Monaco, in: Fundamentals of Crystallography, Ed. C. Giacovazzo for the IUCr, Oxford Scicnce Publications, Oxford 1992, Ch. 4.

[3] M.J. Cooper, R. Natlians, Acta Crystallogr. A 24, 619, 624 (1968).

[4] J.R. Einstcin, J. Appl. Crystallogr. 7, 331 (1974).

[5] B.P. Schoenborn, Acta Crystallogr. A 39, 315 (1983).

[6] F.S. Nielsen, P. Lee, P. Coppens, Acta Crystallogr. A 42, 359 (1986).

[7] P. Coppens, Synchrotron Radiation Crystallography, Academic Press, 1992.

[8] F. Smend, D. Schaupp, II. Czerwinski, A.II. Millhause, II. Schenk-Strauss, DESY Internal Report No. SR-84-003, DESY/IIASYLAB, Hamburg 1984.

[9] A. Kirfel, K. Eichhorn, Acla Crystallogr. A 46, 271 (1990).

[10] D.R. Chipman, Acta Crystallogr. A 25, 209 (1969).

[11] U.W. Arndt, J. Phys. E 11, 671 (1978).

[12] K. Eichhorn, DIF 4 User's Ginide, Revision 2.1, HASYLAB at DESY, Hamburg 1993, unpublished.

[13] M. Wendschuht-Josties, R. Wulf, J. Appl. Crystallogr. 22, 382 (1989).

[14] Y. Anemiya, Synchrolion Radiat. News 3, 21 (1990).

[15] U.W. Arndt, A.J. Wonacott, The Rotation Method in Crystallography, North-IIolland, Ainsterdam, 1977. 